\title{
RELACIÓN ESPACIAL ENTRE LA CONDUCTIVIDAD ELÉCTRICA Y ALGUNAS PROPIEDADES QUÍMICAS DEL SUELO
}

\section{SPATIAL RELATION BETWEEN ELECTRICAL CONDUCTIVITY AND SOME CHEMICAL SOIL PROPIERTIES}

\author{
Diego L. Cortés-D. ${ }^{1}$; Jhon H. Pérez-B. ${ }^{2}$; Jesús H. Camacho-Tamayo ${ }^{3}$
}

\begin{abstract}
${ }^{1}$ Ingeniero Agrícola, Estudiante de Maestría en Ingeniería Agrícola, CR 3045 03, Universidad Nacional de Colombia, Bogotá, Colombia, E-mail: dlcortesd@unal.edu.co; ${ }^{2}$ Estudiante de Ingeniería Agrícola, CR 3045 03, Universidad Nacional de Colombia, Bogotá, Colombia, E-mail: jhperezb@unal.edu.co; ${ }^{3}$ Ingeniero Agrícola, M.Sc., Ph.D. Profesor Asistente. Facultad de Ingeniería, Programa de Ingeniería Agrícola, Universidad Nacional de Colombia. Cra. 45 No. 45 - 03, Bogotá, Colombia. E-mail: jhcamachot@unal.edu.co *Autor para correspondencia.
\end{abstract}

Rev. U.D.C.A Act. \& Div. Cient. 16(2): 401-408, Julio-Diciembre, 2013

\section{RESUMEN}

El conocimiento de los suelos agrícolas resulta ser el factor predominante para el desarrollo óptimo de las actividades agropecuarias. El objetivo de esta investigación fue determinar la relación entre la conductividad eléctrica y las propiedades químicas del suelo en un Andisol. En un área de 83 hectáreas, se realizó un muestreo por medio de una grilla regular rígida geo-referenciada de 80 x $80 \mathrm{~m}$, con el fin de evaluar la variabilidad espacial de las propiedades. Las propiedades medidas fueron $\mathrm{pH}$, conductividad eléctrica (CE), contenido de agua, $\mathrm{Na}, \mathrm{Mg}$ y $\mathrm{Ca}$. El pH presentó una baja variabilidad representada, con un $\mathrm{CV}$ de $6,95 \%$. El contenido de agua, Na y $\mathrm{Mg}$ presentaron variabilidad media, con CV de $26,33 \%, 40,14 \%$ y $57,53 \%$, respectivamente, mientras la variabilidad alta corresponde a las variables de CE y Ca, con un CV de $69,86 \%$ y $74,36 \%$, respectivamente. Con la información obtenida, se realizaron mapas de contorno de cada variable, verificando alta correlación espacial, entre CE y los cationes. El catión con mayor influencia y correlación con CE fue Na con un nivel de significancia del $99 \%$, por lo cual, se elaboró un modelo lineal entre conductividad y sodio, cuyo coeficiente de determinación fue de 0,79. La correlación de Pearson entre la conductividad eléctrica y los contenidos de Ca y Mg fue baja y no significativa, situación que permite concluir que dichos elementos no afectan la CE y, por tanto, el modelo encontrado entre el Na y la CE puede ser aplicado, aun cuando se usen enmiendas en este suelo.

Palabras clave: Semivariograma, distribución espacial, conductividad eléctrica, kriging.

\section{SUMMARY}

Knowledge of agricultural soils results in the predominant factor for an optimal development of agronomic activities. The objective of this work was to determine the relationship between electrical soil conductivity and some chemical properties in an Andisol. An area of 83 hectares was sampled by a rigid regular georeferenced grid of $80 \times 80 \mathrm{~m}$ in order to evaluate the spatial variability of the properties. The properties measured were: $\mathrm{pH}$, electrical conductivity (EC), moisture content, $\mathrm{Na}, \mathrm{Mg}$ and $\mathrm{Ca}$. The $\mathrm{pH}$ showed a low variability with a coefficient of variation (CV) of $6,95 \%$; the moisture content, $\mathrm{Na}$ and $\mathrm{Mg}$ presented a medium variability evidencing, respectively, a CV of $26,33 \%, 40.14 \%$ and $57.53 \%$. A high variability corresponded to the EC and Ca, showing CVs of $69.86 \%$ and $74.36 \%$, respectively. With this information contour maps were produced for each variable, finding a remarkable spatial relationship between EC and soil cations. The most influential one and correlated cation with EC was Na with a significance level of $99 \%$; therefore a linear model between conductivity and sodium was developed, obtaining a coefficient of determination of 0.79 . The Pearson correlation between electrical conductivity and the contents of $\mathrm{Ca}$ and $\mathrm{Mg}$ was low and not significant, situation that allowed to infer that these elements do not affect the EC. Therefore, the model established between Na and EC may even be adapted when amendments are used in this soil.

Key words: Semivariogram, spatial distribution, electrical conductivity, kriging. 


\section{INTRODUCCIÓN}

La base de la agricultura de precisión es el conocimiento de la variabilidad espacial de algunos factores de suelo y su relación con la producción, concepción fundamental para establecer sistemas de producción con mayor sostenibilidad y de mayor eficiencia (Valbuena et al. 2008), pues la producción de los cultivos se ve influenciada por la variación espacial de algunos factores del suelo (Godwin \& Miller, 2003).

En la actualidad, los suelos agrícolas enfrentan serios problemas que afectan, directamente, el desarrollo de los cultivos, los cuales, se relacionan, entre otros, con el contenido de sales, la acidez y la cantidad de nutrientes disponibles. La salinidad del suelo en exceso afecta la productividad agrícola, además de causar la degradación del suelo. Esto, sumado a los graves problemas de contaminación originados por prácticas agrícolas inadecuadas, provocan una disminución en el desarrollo y la producción de diferentes cultivos, lo cual, trae repercusiones a nivel socio-económico, sobre todo en aquellos países que tienen un marcado enfoque agrícola (Rueda et al. 2011).

El control y el manejo apropiado de los diversos tipos de suelos permite el aprovechamiento eficiente y seguro de los insumos. Esto se puede llevar a cabo mediante técnicas, como la agricultura de precisión, que presenta nuevas tecnologías para el manejo de cultivos (Molin et al. 2008) y que, a su vez, satisface el concepto de agricultura sostenible, que se basa en la búsqueda del equilibrio entre la maximización de la productividad de cultivos y la estabilidad económica, reduciendo al mínimo, tanto la utilización de recursos naturales como el impacto ambiental (Corwin et al. 1999).

A través de la medición de ciertas características del suelo, como es el caso de la conductividad eléctrica (CE) y su posterior mapeo, se permite establecer la factibilidad, la viabilidad y el buen desarrollo de un producto en un suelo específico, ya que se considera un método rápido y económico de indicar su productividad (McBride et al. 1990). Además, la CE se ve influenciada por el contenido de agua, el de arcilla y la presencia de iones intercambiables en el suelo (Corwin et al. 1999), capaces de conducir la corriente eléctrica y que inciden en las características nutritivas del suelo. Estas propiedades están claramente asociadas con la salinidad del suelo y su estimación y predicción espacial representan un especial interés científico, para nuevas aplicaciones agrícolas o ambientales (Allaire et al. 2012).

De acuerdo con Parr et al. (1992), diferentes atributos químicos, físicos y biológicos interactúan de manera compleja y su entendimiento es esencial para favorecer la sostenibilidad y mejorar la capacidad productiva del suelo. Tales atributos varían en el espacio y en el tiempo, donde algunos atribu- tos no pueden ser medidos intensivamente de forma rápida, sencilla o a bajo costo, siendo la geoestadística una herramienta para hacer frente a esta situación, para optimizar las distancias de muestreo y definir las variaciones espaciales de los atributos (Juan et al. 2011).

Por las razones expuestas, el objetivo de este estudio fue establecer la relación espacial entre la conductividad eléctrica y algunas propiedades químicas del suelo, en un Andisol del Centro Agropecuario Marengo.

\section{MATERIALES Y MÉTODOS}

El estudio, se realizó en el Centro Agropecuario Marengo (CAM) de la Universidad Nacional de Colombia, ubicado en el municipio de Mosquera (Cundinamarca), en las coordenadas geográficas $4^{\circ} 42^{`} \mathrm{~N}, 74^{\circ} 12^{`} \mathrm{~W}$ y altitud de $2543 \mathrm{~m}$. La zona presenta temperatura media anual de $13,1^{\circ} \mathrm{C}$ y precipitación media anual 670mm (Carranza et al. 2008); pertenece a la cuenca hidrográfica del río Bogotá, que presenta suelos de la serie Marengo y corresponde a la unidad taxonómica Typic Melanudand, con texturas franco limosas y franco arcillosas y con pendientes inferiores al 1\%. El suelo es un Andisol con un horizonte A espeso y oscuro, originado de sedimentos lacustres, con aspersiones de cenizas volcánicas y aportes de arcillas aluviales (Malagón, 2003), con un drenaje natural entre pobre e imperfecto, problemas de salinidad y bajos contenidos de sodio.

En un área cercana de 90ha, se estableció una red rígida de 80m de largo, obteniendo 120 puntos de muestreo, el cual, se realizó entre el primero y el 5 de mayo de 2012. Cada punto fue geo-referenciado, utilizando el receptor GPS Garmin $60 \mathrm{CS} x$ y se tomaron cerca de $250 \mathrm{~g}$ de suelo en los primeros $20 \mathrm{~cm}$ de profundidad, en cada punto.

Las pruebas físico-químicas del suelo, se realizaron sobre muestras secadas por un periodo de 48 horas, a $35^{\circ} \mathrm{C}$, a partir de las cuales, se determinaron el $\mathrm{pH}$, mediante potenciómetro; la conductividad eléctrica (CE), mediante la metodología de extracto de pasta de saturación y lectura en conductímetro y $\mathrm{Na}, \mathrm{Mg}$ y Ca, mediante absorción atómica. De acuerdo con Friedman (2005), estos elementos corresponden a atributos relevantes de la solución del suelo, de donde se define la relación de absorción de sodio en suelos con $\mathrm{pH}$ altos, así como relacionados directamente con la capacidad de intercambio catiónica. Se consideró realizar una corrección de la conductividad eléctrica por el contenido de agua de las muestras, a partir del modelo empírico propuesto por Vogeler \& Clothier (1996), dado que varios autores coinciden en que este modelo permite un buen ajuste (Abbasi et al. 2006; Magesan et al. 2003). El modelo es definido por la siguiente ecuación: 


$$
\sigma=2,5667 \times \theta \times \sigma_{\mathrm{w}}
$$

(Ecuación1)

donde $\theta$ es el contenido de agua y $\sigma_{\mathrm{w}}$ es la conductividad eléctrica en $\mathrm{dS} \mathrm{m}^{-1}$. El factor 2,5667, se determinó mediante el uso de la herramienta SOLVER de Excel.

Mediante el paquete SPSS v. 20, se realizó el análisis estadístico, en el que se evaluaron y se descartaron los datos atípicos, mediante el análisis del histograma y de diagramas box-plot. Además, se determinaron los estadísticos descriptivos de las variables estudiadas, para poder apreciar su comportamiento y su distribución. La variabilidad, se evaluó con base en el coeficiente de variación (CV) que, de acuerdo con Warrick \& Nielsen (1980), atributos con CV menor al 12\%, presenta baja variabilidad; media, entre $12 \%$ y $60 \%$ y para parámetros con alta variabilidad, se tiene coeficientes de variación superiores al $60 \%$. Igualmente, se estudió la relación entre propiedades del suelo, a través de la correlación de Pearson, con el fin de observar el catión con mayor influencia en la conductividad eléctrica y, de esta manera, establecer un modelo matemático, que permitiese especificar, de forma rápida y económica, la concentración de tal catión, mediante la medición de la CE.

Adicionalmente, se evaluó la dependencia espacial de las propiedades en estudio, a través de la teoría de variables regionalizadas, la cual, dispone de diferentes métodos de análisis de variación espacial, siendo una de ellas el semivariograma (Webster \& Oliver, 2007). A partir del ajuste de los modelos, se determinó la pepita $\left(\mathrm{C}_{0}\right)$, la meseta $\left(\mathrm{C}_{0}+\mathrm{C}\right)$ y el rango o alcance $(\mathrm{A})$, además del grado de dependencia espacial (GDE), como la razón entre la pepita y la meseta $\left(C / C_{0}+C\right)$, siendo considerada fuerte para GDE, superior de 0,75; moderada para GDE, entre 0,25 y 0,75 y débil para GDE, inferior a 0,25 (Cambardella et al. 1994). Los semivariogramas, se calcularon con el programa GS+ v.9 (Gamma Design Software).

Para la selección de los modelos teóricos de semivariograma, se consideró el menor valor de la suma de los cuadrados residuales, el coeficiente de determinación $\left(R^{2}\right)$ de la ecuación de ajuste y los valores semejantes, obtenidos entre el valor real y el valor estimado, que se obtienen en la validación cruzada, indicadores apropiados, para tal fin (Garzón-Gutiérrez et al. 2010). A partir de estos resultados, se elaboraron mapas de distribución espacial, usando el programa Surfer 10.0 (Golden Software Inc., Golden, CO), mediante kriging ordinario, para cada una de las variables.

\section{RESULTADOS Y DISCUSIÓN}

Los resultados obtenidos, a partir del análisis de estadística descriptiva, permiten verificar que se cuenta con distribuciones simétricas, con valores de media y mediana, similares para cada profundidad analizada (Tabla 1). Si bien los valores de asimetría y curtosis para Na y Mg son distantes de cero, alejándose del comportamiento normal, es conveniente verificar que la distribución normal no presente colas largas, que comprometan los resultados (Cressie, 1993), especialmente, cuando se realiza kriging, donde las estimaciones son basadas en valores medios (Warrick \& Nielsen, 1980). Otro factor significativo es la ocurrencia del efecto proporcional entre la media y la varianza de los datos en el espacio, para estimar mesetas bien definidas, en los modelos teóricos de semivariogramas.

Los valores de las propiedades en estudio, se encuentran en los rangos reportados para andisoles, por Garzón-Gutiérrez et al. (2010) y Sánchez et al. (2011), con medias inferiores, debido a la alta precipitación ocasionada por el fenómeno de La Niña, que ocasionó un lavado de los cationes, debido a la presencia de un nivel freático superficial, por periodos mayores a los usualmente registrados para la zona, impidiendo también el uso del suelo de forma adecuada. Juan et al. (2011) indican que la alta precipitación esta aparentemente asociada a las bajas concentraciones de estos elementos, debido a la posible lavado de los horizontes superiores.

De acuerdo con los estadísticos descriptivos, se observa que la menor variabilidad la presentó el $\mathrm{pH}(\mathrm{CV}=6,95 \%)$, comportamiento típico para esta propiedad, en diferentes tipos de suelos (Cruz et al. 2011; Garzón-Gutiérrez et al. 2010;

Tabla 1. Estadísticos descriptivos del contenido de agua $\left(\mathrm{C}_{\mathrm{A}}\right), \mathrm{pH}$, conductividad eléctrica (CE), Na, $\mathrm{Mg}$ y Ca.

\begin{tabular}{|c|c|c|c|c|c|c|c|}
\hline Propiedad & Media & Mediana & C.V. \% & Mínimo & Máximo & Asimetría & Curtosis \\
\hline $\mathrm{C}_{\mathrm{A}}\left(\mathrm{g} \mathrm{g}^{-1}\right)$ & 0,369 & 0,348 & 26,33 & 0,254 & 0,709 & 1,84 & 3,71 \\
\hline $\mathrm{pH}$ & 5,870 & 5,810 & 6,95 & 5,000 & 6,700 & 0,26 & $-0,56$ \\
\hline $\mathrm{CE}\left(\mathrm{dS} \mathrm{m}^{-1}\right)$ & 0,014 & 0,012 & 69,86 & 0,003 & 0,043 & 1,34 & 1,39 \\
\hline $\mathrm{Na}\left(\mathrm{mg} \mathrm{g}^{-1}\right)$ & 0,504 & 0,466 & 40,14 & 0,240 & 1,286 & 2,03 & 5,68 \\
\hline $\mathrm{Mg}\left(\mathrm{mg} \mathrm{g}^{-1}\right)$ & 0,734 & 0,637 & 57,53 & 0,269 & 2,759 & 2,88 & 11,95 \\
\hline $\mathrm{Ca}\left(\mathrm{mg} \mathrm{g}^{-1}\right)$ & 1,989 & 1,746 & 74,36 & 0,245 & 5,833 & 0,63 & $-0,25$ \\
\hline
\end{tabular}


Najafian et al. 2012). El contenido de agua y la concentración de Na y Mg presentaron variabilidad media (Tabla 1). La alta variabilidad observada para CE y la concentración de $\mathrm{Ca}$, no es comúnmente reportada por diferentes autores, pues es señalado que estas propiedades presentan, generalmente, baja o media variabilidad (Sánchez et al. 2011; Najafian et al. 2012). La variabilidad de estas propiedades puede ser natural, debida, especialmente, al relieve, material parental y condiciones climáticas o inducida por diferentes actividades en la zona de estudio, es decir, manejo de diferentes cultivos (maíz, hortalizas, papa, frutales, entre otros) y ganadería. Wei et al. (2009) atribuyen el comportamiento de altas variaciones a aplicaciones desiguales de enmiendas o fertilizantes e ineficacia de las prácticas de manejo sobre el terreno.

Se identificaron dos datos atípicos. En el caso 55, la variable motivo de anomalía fue el sodio, debido a su mayor concentración, respecto a los otros puntos de muestreo (Tabla 2); sin embargo, al observarse las demás variables, en estas se encuentran valores que no se alejan de la media, razón por la cual, esta propiedad es descartada para el análisis de dependencia espacial de la misma. Para el caso 57, se observa una alta concentración de magnesio, a la vez, que la conductividad eléctrica y las concentraciones de sodio y de calcio son igualmente altas, por lo tanto, se conserva este punto del muestreo. La tabla 3 muestra la matriz de correlación de Pearson obtenida.

De acuerdo a la matriz de correlación, se observa que el catión que más incide en la conductividad eléctrica es el sodio, con un coeficiente de correlación de 0,59 y un nivel de significancia del $99 \%$. Este comportamiento, se confirma en el modelo exponencial ajustado, donde se observa que el contenido de Na del suelo se puede obtener a partir de CE (Figura 1), a pesar de la alta dispersión encontrada, debido a los bajos valores de CE y de los contenidos de Na.

Posteriormente, se verificó la dependencia especial de cada propiedad analizada, obteniendo los parámetros geoestadísticos (Tabla 4). De acuerdo con el criterio de Cambardella et

Tabla 2. Valores de las propiedades en las muestras atípicas.

\begin{tabular}{|l|r|r|}
\hline \multicolumn{1}{|c|}{ Caso } & \multicolumn{2}{|c|}{55} \\
\hline Variable de motivo & $\mathrm{Na}$ & $\mathrm{Mg}$ \\
\hline Impacto de la variable & 0,531 & 0,326 \\
\hline Valor de la variable & 1,120 & 2,760 \\
\hline Norma de la variable & 0,442 & 0,805 \\
\hline $\mathrm{C}_{\mathrm{A}}\left(\mathrm{g} \mathrm{g}^{-1}\right)$ & 0,370 & 0,460 \\
\hline $\mathrm{pH}$ & 6,200 & 6,570 \\
\hline $\mathrm{CE}\left(\mathrm{dS} \mathrm{m}^{-1}\right)$ & 0,005 & 0,040 \\
\hline $\mathrm{Na}\left(\mathrm{mg} \mathrm{g}^{-1}\right)$ & 1,116 & 1,286 \\
\hline $\mathrm{Mg}\left(\mathrm{mg} \mathrm{g}^{-1}\right)$ & 1,242 & 2,759 \\
\hline $\mathrm{Ca}\left(\mathrm{mg} \mathrm{g}^{-1}\right)$ & 4,510 & 1,426 \\
\hline
\end{tabular}

Tabla 3. Correlación de Pearson del contenido de agua $\left(\mathrm{C}_{\mathrm{A}}\right), \mathrm{pH}$, conductividad eléctrica (CE), Na, $\mathrm{Mg}$ y Ca.

\begin{tabular}{|l|c|c|c|c|c|c|}
\hline & $\mathbf{C}_{\mathrm{A}}$ & $\mathbf{p H}$ & $\mathbf{C E}$ & Na & $\mathbf{M g}$ & $\mathbf{C a}$ \\
\hline $\mathbf{C}_{\mathrm{A}}$ & 1 & & & & & \\
\hline $\mathbf{p H}$ & 0,030 & 1 & & & & \\
\hline $\mathrm{CE}$ & $0,423^{* *}$ & 0,030 & 1 & & & \\
\hline $\mathrm{Na}$ & $0,362^{*}$ & 0,070 & $0,590^{* *}$ & 1 & & \\
\hline $\mathbf{M g}$ & 0,280 & 0,210 & 0,300 & $0,641^{* *}$ & 1 & \\
\hline $\mathrm{Ca}$ & 0,070 & $-0,240$ & $-0,010$ & $-0,130$ & $0,331^{*}$ & 1 \\
\hline
\end{tabular}

*La correlación es significante al nivel 95\%; **La correlación es significativa al nivel 99\%. 
al. (1994), el calcio presentó una fuerte dependencia espacial, mientras el $\mathrm{pH}$ y el $\mathrm{Mg}$ expresaron una moderada dependencia, con valores cercanos a 0,5.

En cuanto al rango, se encuentra que el magnesio y el calcio presentan los mayores valores y, de manera simultánea, el sodio exhibe un rango significativamente bajo. Caso contrario el $\mathrm{pH}$, el cual, presenta uno de los rangos más bajos y que acompañado de un $\mathrm{R}^{2}$, dependencia espacial y un coeficiente de validación cruzada (CVC), significativamente bajos, se infiere que los datos obtenidos no son suficientemente representativos (Medina et al. 2012).

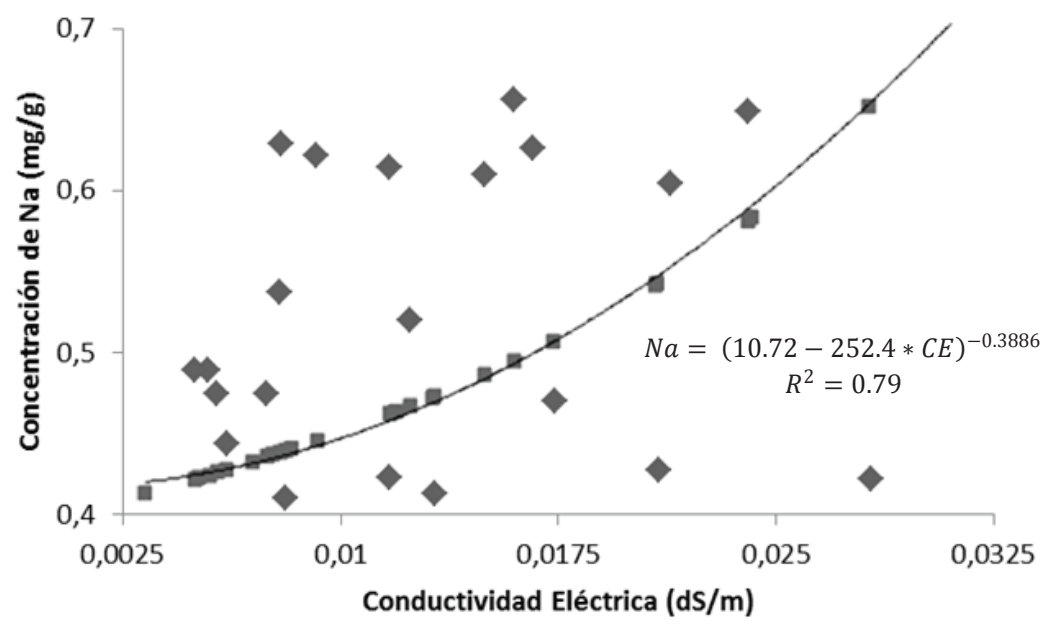

Figura 1. Modelo obtenido para la determinación de Na, a partir de la conductividad eléctrica.

El calcio fue el que mayor CVC presentó, lo cual, representa que los valores estimados, según el modelo encontrado, se ajustan muy bien a los datos reales, soportado por un elevado coeficiente de determinación y una fuerte dependencia espacial, mientras que los menores valores de CVC, se presentaron en el pH y en el Magnesio.

En concordancia con Morales et al. (2009), a menudo se registra que los modelos que mejor se ajustan al $\mathrm{pH}$ son los esféricos. De igual manera, la CE también reflejó ajuste a un modelo esférico, como también es reportado por Heil \&
Schmidhalter (2012). El ajuste de una propiedad a un modelo de semivariograma muestra la aleatoriedad, homogeneidad o tendencia espacial de ésta, que depende de factores naturales o inducidos, por lo cual, su distribución en el espacio depende de condiciones edafoclimáticas en el tiempo o por la acción del hombre, conduciendo, incluso, a diferentes modelos espaciales en periodos relativamente cortos, en el estudio de una propiedad en la misma zona (Li et al. 2007).

Al encontrarse un rango alto en el magnesio y en el calcio, se puede inferir que este es resultado de la aplicación con

Tabla 4. Parámetros de los modelos ajustados de semivariogramas para contenido de agua $\left(\mathrm{C}_{\mathrm{A}}\right)$, $\mathrm{pH}$, conductividad eléctrica (CE), Na, Mg y Ca.

\begin{tabular}{|c|c|c|c|c|c|c|c|}
\hline Propiedad & Modelo & Co & Co $+\mathbf{C}$ & Rango, $\mathbf{m}$ & $\mathbf{R}^{2}$ & C/(Co+C) & CVC \\
\hline $\mathrm{C}_{\mathrm{A}}$ & Esférico & 0,001 & 0,007 & 515,0 & 0,722 & 0,814 & 0,756 \\
\hline $\mathrm{pH}$ & Esférico & 0,066 & 0,156 & 404,0 & 0,774 & 0,577 & 0,685 \\
\hline $\mathrm{CE}$ & Esférico & $7,5 \mathrm{E}-6$ & $5,69 \mathrm{E}-5$ & 414,0 & 0,787 & 0,868 & 0,877 \\
\hline $\mathrm{Na}$ & Exponencial & $1,59 \mathrm{E}-4$ & 0,014 & 264,0 & 0,405 & 0,887 & 0,822 \\
\hline $\mathrm{Mg}$ & Gaussiano & 0,035 & 0,078 & 800,2 & 0,657 & 0,548 & 0,658 \\
\hline $\mathrm{Ca}$ & Gaussiano & 0,330 & 3,670 & 1269,6 & 0,964 & 0,910 & 1,006 \\
\hline
\end{tabular}




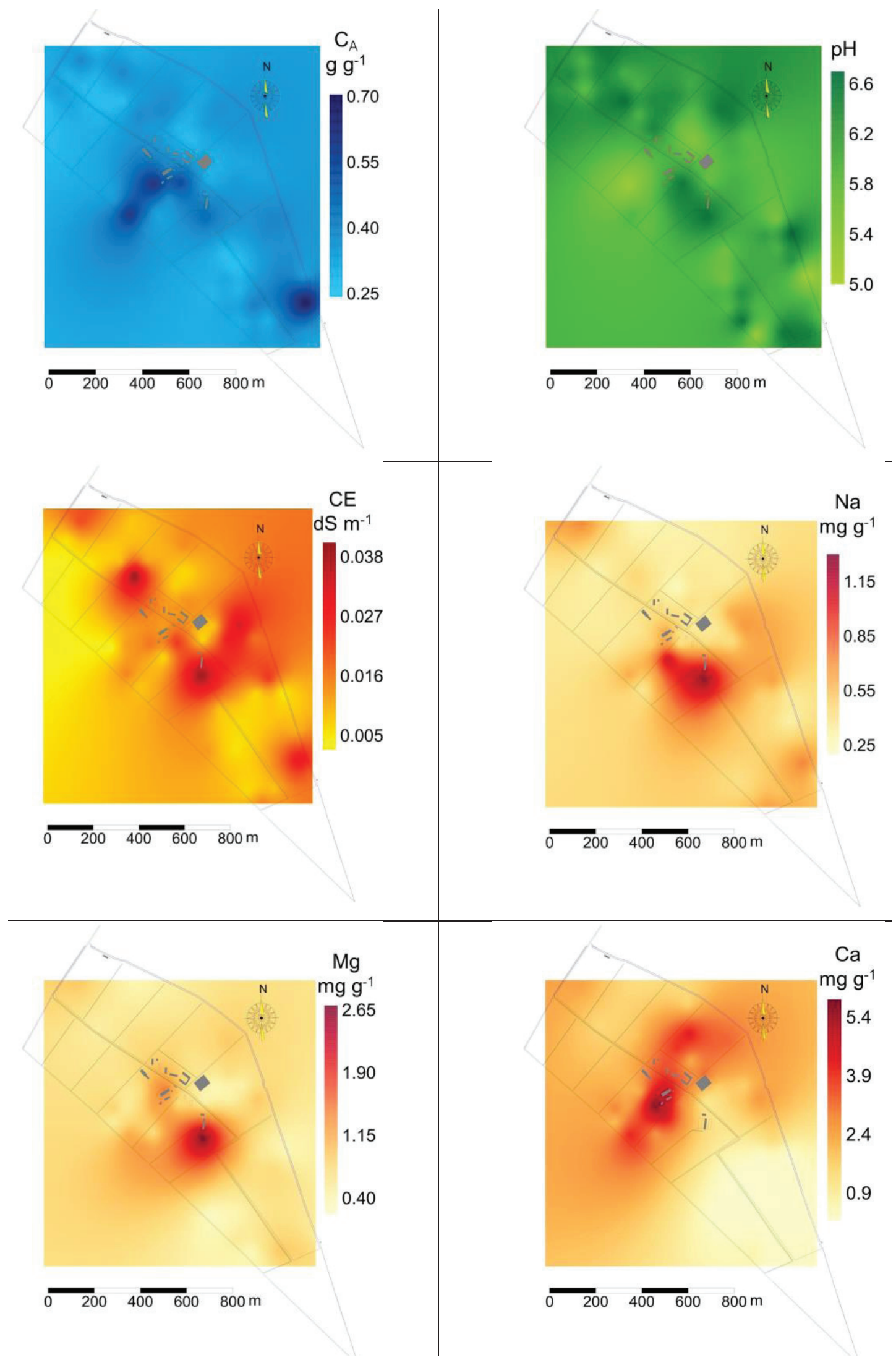

Figura 2. Mapas de distribución del contenido de agua (A), pH (B), conductividad eléctrica (C), $\mathrm{Na}(\mathrm{D}), \mathrm{Mg}(\mathrm{E})$ y Ca (F) (sobrepuesto al mapa de la localización de la zona de estudio). 
cal dolomita en el manejo de pasturas y en la producción agrícola, al ser estos cationes los predominantes del suelo, aun cuando la dependencia espacial del magnesio se hace moderada.

La obtención de coeficientes de validación cruzada cercanos a uno para el sodio y la conductividad eléctrica, se valida la confiabilidad de los datos usados para la construcción del modelo, que correlaciona estos dos atributos, además de que presentan una fuerte dependencia espacial, de acuerdo a la clasificación dada por Cambardella et al. (1994).

Observando los mapas, se encuentra que la correlación de la conductividad eléctrica y la concentración de sodio también se evidencian espacialmente (Figura 2), proporcionando mayor validez del modelo de correlación registrado, lo que permite, de manera fácil, rápida y económica, tomar decisiones inmediatas, para las labores de manejo y de fertilización.

En general, se observa también relación espacial de la conductividad eléctrica junto con los cationes analizados, donde zonas con altos valores de CE, corresponden con zonas de altos contenidos de $\mathrm{Ca}, \mathrm{Mg}$ y $\mathrm{Na}$.

Esta metodología permitió analizar la CE, su relación con otras propiedades del suelo, donde los contenidos de Na presentan una relación directa y significativa con esta propiedad, relación que es verificada en la obtención del modelo exponencial, así como en la correlación lineal y en la correspondencia visual entre los mapas de estas propiedades del suelo. De igual manera, es conveniente considerar la variabilidad espacial de estas propiedades para el manejo localizado, que en el futuro se realice en el manejo de pasturas y de cultivos.

Conflicto de intereses: El manuscrito fue preparado y revisado con la participación de todos los autores, quienes declaramos que no existe ningún conflicto de intereses que ponga en riesgo la validez de los resultados presentados.

\section{BIBLIOGRAFÍA}

1. ABBASI, F.; JAVAUX, M.; VANCLOOSTER, M.; FEYEN, J.; WYSEURE, G.; NZIGUHEBA, G. 2006. Experimental study of water flow and sulphate transport at monolith scale. Agric. Water Manag. 79(1):93-112.

2. ALLAIRE, S.E.; LANGE, S.F.; LAFOND, J.A.; PELLETIER, B.; CAMBOURIS, A.N.; DUTILLEUL, P. 2012. Multiscale spatial variability of $\mathrm{CO}_{2}$ emissions and correlations with physico-chemical soil properties. Geoderma (Netherlands). 170:251-260.

3. CAMBARDELLA, C.A.; MOORMAN, T.B.; NOVAK, J.M.; PARKIN, T.B.; KARLEN, D.L.; TURCO, R.F.; KONO-
PKA, A.E. 1994. Field-scale variability of soil properties in Central Iowa Soils. Soil Sci. Soc. Am. J. 58(5):1501-1511.

4. CARRANZA, C.; LANCHERO, O.; MIRANDA, D.; SALAZAR, M.R.; CHAVES, B. 2008. Modelo simple de simulación de distribución de masa seca en brócoli (Brassica sp.) variedad Coronado y repollo (Brassica oleracea) híbrido Delus cultivados en la Sabana de Bogotá. Agron. Col. 26(1):23-31.

5. CORWIN, D.L.; LOAGUE, K.; ELLSWORTH, T.R. 1999. Introduction: Assessing non-point source pollution in the vadose zone with advanced information technologies. In: Corwin, D.L.; Loague, K.; Ellsworth, T.R. eds. Assessment of Nonpoint Source Pollution in the Vadose Zone. Geophysical Monograph Series. Vol. 108. AGU, Washington, D.C., USA, p.120.

6. CRESSIE, N. 1993. Statistics for spatial data, John Wiley \& Sons (New York). 928p.

7. CRUZ, J.S.; ASSIS JUNIOR, R.N.; MATIAS, S.S.R.; CAMACHO-TAMAYO, J.H. 2011. Spatial variability of an Alfisol cultivated with sugarcane. Cienc. Inv. Agr. (Chile). 38(1):155-164.

8. FRIEDMAN, S.P. 2005. Soil properties influencing apparent electrical conductivity: a review. Comp. Electron. Agric. (USA). 46(1-3):45-70.

9. GARZÓN-GUTIÉRREZ, C.A.; CORTÉS, C.A; CAMACHO-TAMAYO, J.H. 2010. Variabilidad espacial de algunas propiedades químicas de un entisol. Rev. U.D.C.A Act. \& Div. Cient. (Colombia). 13(1):87-95.

10. GODWIN, R.; MILLER, C. 2003. Review of the technologies for mapping within-field variability. Biosys. Eng. (United Kingdom). 84(4):393-407.

11. HEIL, K.; SCHMIDHALTER, U. 2012. Characterisation of soil texture variability using the apparent soil electrical conductivity at a highly variable site. Comput. Geosci. (USA). 39:98-110.

12. JUAN, P.; MATEU, J.; JORDAN, M.M.; MATAIX-SOLERA, J.; MELÉNDEZ-PASTOR, I.; NAVARRO-PEDREÑO, J. 2011. Geostatistical methods to identify and map spatial variations of soil salinity. J. Geochem. Explor. (USA). 108(1):62-72.

13. LI, Y.; SHI, Z.; LI, F. 2007. Delineation of Site-Specific Management Zones Based on Temporal and Spa- 
tial Variability of Soil Electrical Conductivity. Pedosphere (China). 17(2):156-164.

14. MAGESAN, G.N.; VOGELER, I.; CLOTHIER, B.E.; GREEN, S.R.; LEE, R. 2003. Solute Movement through an Allophanic Soil. J. Environ. Qual. (USA). 32(6):2325-2333.

15. MALAGÓN, D. 2003. Ensayo sobre tipología de suelos colombianos - Énfasis en génesis y aspectos ambientales. Rev. Acad. Col. Cienc. Exact. Fis. Nat. 27(104):319-341.

16. McBRIDE, R.A.; GORDON, A.M.; SHRIVE, S.C. 1990. Estimating forest soil quality from terrain measurements of apparent electrical conductivity. Soil Sci. Soc. Am. J. 54(1):290-293.

17. MEDINA, B.C.; CAMACHO-TAMAYO, J.H.; CORTES, C.A. 2012. Soil penetration resistance analysis by multivariate and geostatistical methods. Eng. Agríc. (Brasil). 32(1):91-101.

18. MOLIN, J.P.; NUNES, C. 2008. Establishing management zones using soil electrical conductivity and other soil properties by the fuzzy clustering technique. Sci. Agric. (Brasil). 65(6):565-573.

19. MORALES, L.A.; PAZ FERREIRO, J. 2009. Geostatistical analysis of $\mathrm{pH}$ and redox potential (Eh) variability over a rice field in successive crop stages. En: Silva, O.; Carrera, J. (eds.) Estudios en la zona no saturada del suelo. Vol IX, 9p.

20. NAJAFIAN, A.; DAYANI, M.; MOTAGHIAN, H.R.; NADIAN, H. 2012. Geostatistical Assessment of the Spatial Distribution of Some Chemical Properties in Calcareous Soils. J. I. A. (China). 11(10):1729-1737.

21. PARR, J.F.; HORNICK, S.B.; PAPENDICK, R.I. 1992. Soil quality: attributes and relationship to alterna- tive and sustainable agriculture. Amer. J. Altern. Agric. 7(1-2):5-11.

22. RUEDA SAA, G.; RODRÍGUEZ VICTORIA, J.A.; MADRINAN MOLINA, R. 2011. Metodologías para establecer valores de referencia de metales pesados en suelos agrícolas: perspectivas para Colombia. Acta Agron. 60(3):203-217.

23. SÁNCHEZ T., J.D.; LIGARRETO, G.A.; LEIVA, F.R. 2011. Spatial variability of soil chemical properties and its effect on crop yields: a case study in maize (Zea mays L.) on the Bogota Plateau. Agron. Col. 29(2):456-466.

24. VALBUENA, C.A.; MARTÍNEZ, L.J.; GIRALDO, R. 2008. Variabilidad espacial del suelo y su relación con el rendimiento de mango (Mangifera indica L.). Rev. Bras. Frutic. (Brasil). 30(4):1146-1151.

25. VOGELER, I.; CLOTHIER, B.E. 1996. Characterizing water and solute movement by TDR and disk permeametry. Soil. Sci. Soc. Am. J. 60(1):5-12.

26. WARRICK, A.W.; NIELSEN, D.R. 1980. Spatial variability of soil physical properties in field. In: Hillel, D. (ed). Applications of soil physics. Academic Press (New York). p.319-344.

27. WEBSTER, R.; OLIVER, M.A. 2007. Geostatistics for environmental scientists. John Wiley \& Sons Inc., Hoboken, NJ. 332p.

28. WEI, Y.; BAI, Y.; JIN, J.; ZHANG, F.; ZHANG, L.; LIU, X. 2009. Spatial variability of soil chemical properties in the reclaiming marine foreland to yellow sea of china. Agr. Sci. China. 8(9):1103-1111.

Recibido: Noviembre 29 de 2012

Aceptado: Agosto 26 de 2013

Como citar:

Cortés-D., D.L.; Pérez-B., J.H.; Camacho-Tamayo, J.H. 2013. Relación espacial entre la conductividad eléctrica y algunas propiedades químicas del suelo. Rev. U.D.C.A Act. \& Div. Cient. 16(2): 401-408. 